\title{
AGN and Starbursts in Dusty Galaxy Mergers: Insights from the Great Observatories All-sky LIRG Survey
}

\author{
Joseph M. Mazzarella \\ and the GOALS Team
}

\author{
MS 100-22 \\ Infrared Processing \& Analysis Center, California Institute of Technology, \\ Pasadena, CA 91125, USA \\ email: mazz@ipac.caltech.edu
}

\begin{abstract}
The Great Observatories All-sky LIRG Survey (GOALS) is combining imaging and spectroscopic data from the Herschel, Spitzer, Hubble, GALEX, Chandra, and XMM-Newton space telescopes augmented with extensive ground-based observations in a multiwavelength study of approximately 180 Luminous Infrared Galaxies (LIRGs) and 20 Ultraluminous Infrared Galaxies (ULIRGs) that comprise a statistically complete subset of the $60 \mu \mathrm{m}$-selected IRAS Revised Bright Galaxy Sample. The objects span the full range of galaxy environments (giant isolated spirals, wide and close pairs, minor and major mergers, merger remnants) and nuclear activity types (Seyfert 1, Seyfert 2, LINER, starburst/HII), with proportions that depend strongly on the total infrared luminosity. I will review the science motivations and present highlights of recent results selected from over 25 peer-reviewed journal articles published recently by the GOALS Team. Statistical investigations include detection of high-ionization Fe K emission indicative of deeply embedded AGN, comparison of UV and far-IR properties, investigations of the fraction of extended emission as a function of wavelength derived from mid-IR spectroscopy, mid-IR spectral diagnostics and spectral energy distributions revealing the relative contributions of AGN and starbursts to powering the bolometric luminosity, and quantitative structure analyses that delineate the evolution of stellar bars and nuclear stellar cusps during the merger process. Multiwavelength dissections of individual systems have unveiled large populations of young star clusters and heavily obscured AGN in early-stage (II Zw 96), intermediate-stage (Mrk 266, Mrk 273), and late-stage (NGC 2623, IC 883) mergers. A recently published study that matches numerical simulations to the observed morphology and gas kinematics in mergers has placed four systems on a timeline spanning 175-260 million years after their first passages, and modeling of additional (U)LIRGs is underway. A very brief description of ongoing work with Herschel and ALMA will be given. The talk will conclude with a discussion of the demographics of dual AGN (kpc-scale orbits) in the GOALS sample, including the difficulty of their detection and confirmation, a proposed sequence representing a progression from dual AGN to binary AGNs (sub-pc scale orbits), and implications for the scarcity of confirmed binary QSOs in recent large surveys. Grant support from NASA is gratefully acknowledged.
\end{abstract}

Keywords. galaxies: active, galaxies: nuclei, galaxies: peculiar, galaxies: interactions, galaxies: starburst, galaxies: Seyfert, galaxies: structure, galaxies: star clusters, infrared: galaxies

\section{Introduction}

Among the most notable discoveries of the Infrared Astronomical Satellite (IRAS) is a population of extragalactic sources with bolometric luminosities dominated by infrared radiation. Numerous studies (e.g., Sanders \& Mirabel 1996; Veilleux 2006; and references therein) have shown that the most luminous objects, known as Ultraluminous 
Infrared Galaxies (ULIRGs; $\mathrm{L}_{\mathrm{ir}} \geqslant 10^{12} \mathrm{~L}_{\odot}$ ), as well as their less luminous but much more numerous counterparts known as Luminous Infrared Galaxies (LIRGs; $10^{11} \mathrm{~L}_{\odot} \leqslant$ $\mathrm{L}_{\mathrm{ir}}<10^{12} \mathrm{~L}_{\odot}$ ), are fascinating objects with many ramifications for galaxy evolution. They contain the largest rates of star formation; the fraction of detected AGN increases with $L_{i r}$; they typically contain large-scale outflows (superwinds); and evidence has been building that most local (U)LIRGs represent an evolutionary process involving the transformation of major mergers between dusty, gas-rich disk galaxies into elliptical galaxies hosting QSOs or powerful radio galaxies. At redshifts of $\mathrm{z} \sim 1$, LIRGs dominate the total star-formation density (e.g., Le Floc'h et al. 2005), and they have a much higher space density than ULIRGs at that epoch. At $\mathrm{z} \sim 2$ the contributions of LIRGs and ULIRGs to the total IR luminosity density are roughly equal (e.g., Caputi et al. 2007). Since LIRGs and ULIRGs were much more common in the early universe, clearly these populations are fundamental in understanding the co-evolution of star formation and AGN.

Open Science Questions. Local (U)LIRGs are very interesting systems in their own right, and they provide a unique opportunity to study detailed properties that cannot be resolved in their more numerous counterparts at high redshifts. Some of the questions we are investigating include: (1) What are the relative timescales and energetics of active star formation and AGN phenomena during different phases of the merger sequence? (2) Which LIRGs are in a pre-ULIRG (or post-ULIRG) phase of increasing (or decreasing) star formation rates or AGN fueling? (3) Do some LIRGs lack the physical conditions required to elevate $\mathrm{L}_{\mathrm{ir}}>10^{12} \mathrm{~L}_{\odot}$ during a major merger? (4) How do SFRs, AGN activity, and the physical conditions of the dust-emitting regions vary with merger stage, the mass ratio of the galaxies, the geometry of the encounter, and the properties of each galaxy determined from multiwavelength observations?

\section{The Great Observatories All-Sky LIRG Survey}

The Great Observatories All-Sky LIRG Survey (GOALS; http://goals.ipac.caltech.edu/) is utilizing imaging and spectroscopic data from NASA's Spitzer, Hubble, Chandra and GALEX space telescopes, along with ground-based observations, to perform a comprehensive study of more than 200 (U)LIRGs in the local universe (Armus et al. 2009). The GOALS sample consists of 181 LIRGs and 23 ULIRGs that form a statistically complete subset of the flux-limited IRAS Revised Bright Galaxy Sample (RBGS), which itself consists of 629 extragalactic objects with $60 \mu \mathrm{m}$ flux densities $>5.24$ Jy and Galactic latitudes $|b|>5^{\circ}$ (Sanders et al. 2003). Details regarding the data sets are given in Armus et al. (2009). However, since that article was published, the team has extended the survey to include imaging and spectroscopic observations with XMM-Newton, the Herschel Space Observatory, as well as ground-based (sub)millimeter and radio observations with ALMA, EVLA, and the GBT.

In the following sections we briefly review the results of recent survey studies based on X-ray, UV, visual, infrared and radio observations, summarize highlights of detailed multiwavelength investigations of particularly interesting systems, and describe the first results of numerical simulations aimed toward improving our understanding of the starbursts and AGN found in major mergers that are ubiquitous among the most luminous LIRGs and in all ULIRGs. We close with a very brief description of work in progress.

\section{Survey Results}

$X$-rays. The Chandra X-ray Observatory component of GOALS contains 44 (U)LIRGs with $\log \left(\mathrm{L}_{\mathrm{ir}} / \mathrm{L}_{\odot}\right)=11.73-12.57$. In the first results reported, Iwasawa et al. $(2009)$ 
showed that, after omitting 15 obvious AGN with luminous in hard X-rays, the integrated spectrum of the remaining LIRGs that are quiet in hard X-rays shows strong highionization Fe $\mathrm{K}$ emission (Fe xxv at $6.7 \mathrm{keV}$ ). The composite hard X-ray quiet spectrum and the Fe $\mathrm{K}$ feature can be explained by hot gas produced in a starburst only if the accompanying copious emission from high-mass X-ray binaries is somehow suppressed. This implies that deeply embedded supermassive black holes may be the primary power source of their infrared luminosity, and the faint photoionized gas that remains visible is similar to what is observed in some ULIRGs containing Compton-thick AGN. Images, radial brightness distributions, and spectra of the Chandra observations were presented by Iwasawa et al. (2011). Using a selection based on hard X-ray color and the $6.4 \mathrm{keV} \mathrm{Fe}$ line, AGN are found in 37\% of the objects, with a higher AGN fraction found in the higher luminosity sources involved in late-stage mergers. Most of the objects also show extended soft X-ray emission which is likely due to starburst-driven outflows (superwinds).

Infrared Spectroscopy with Spitzer. Initial analysis of mid-IR spectra of 248 LIRGs observed with the Spitzer Infrared Spectrograph (IRS) were presented by Petric et al. (2011). The AGN contribution to the mid-IR emission was estimated via diagnostics involving the $[\mathrm{Ne} \mathrm{v}],[\mathrm{O} \mathrm{IV}]$, and [Ne II] fine-structure emission lines, the $6.2 \mu \mathrm{m} \mathrm{PAH}$ feature, and the shape of the mid-IR continuum. Key results include the finding that $18 \%$ of all LIRGs in the GOALS sample contain an AGN, and in 10\% of the LIRGs the AGN contributes $>50 \%$ of the total infrared luminosity. Summing up the total $\mathrm{L}_{\text {ir }}$ contributed by AGN implies that AGN supply about $12 \%$ of the total energy emitted by LIRGs. In addition, Petric et al. showed that the use of mid-IR broad-band colors to find AGN candidates may miss up to $40 \%$ of AGN-dominated (U)LIRGs. Analysis of the spatial extent of the mid-IR continuum emission in the GOALS sample (Diaz-Santos, T., et al. 2010) shows that although the fraction of extended emission as a function of wavelength varies widely, in about $30 \%$ of the LIRGs at least half of their mid-IR emission is extended, and the compactness of the mid-IR emission increases with increasing total $\mathrm{L}_{\mathrm{ir}}$. In addition, (U)LIRGs that are AGN dominated are more compact, independent of their $\mathrm{L}_{\mathrm{ir}}$ values, and the extent of the mid-IR continuum emission correlates with the global dust temperature. A similar analysis of the spatial extent of mid-IR emission lines by Diaz-Santos et al. (2011) shows that the [Ne II]12.81 $\mu \mathrm{m}$ emission line roughly traces the hot dust continuum, but the PAH emission is significantly more extended. The finding that the 5-14 $\mu \mathrm{m}$ spectra of the extended components are homogeneous for all galaxies in the GOALS sample suggests that the physical properties of star formation occurring farther than $1.5 \mathrm{kpc}$ from the nuclei are very similar and resemble local star-forming galaxies with $\mathrm{L}_{\mathrm{ir}}<10^{11} \mathrm{~L}_{\odot}$, and also resemble ULIRGs dominated by star formation at $\mathrm{z} \sim 2$. In contrast, the mid-IR spectra of the central components are very diverse, implying that observed variations of the integrated properties of local (U)LIRGs reflect processes that are occuring predominantly in their nuclei. The low-resolution IRS spectra spanning 5-58 $\mu \mathrm{m}$ for 244 nuclei in 180 (U)LIRGs in the GOALS sample are presented by Stierwalt et al. (2013), along with the following results: Both silicate depth and mid-IR continuum slope rise with increasing $\mathrm{L}_{\mathrm{ir}}$; on average late-stage mergers have steeper midIR slopes and higher dust obscuration than early and intermediate stage mergers; when AGN contributions to local LIRGs and high-z sub-mm galaxies (SMGs) are removed, there is a strong resemblance between the average local LIRG and SMGs dominated by star formation.

Infrared Spectroscopy with Herschel. First results from observations of the GOALS sample with the PACS instrument onboard the Herschel Space Observatory focused on the [C II] $157.7 \mu \mathrm{m}$ emission line (Diaz-Santos, T., et al. 2013), which is well known to be the primary cooling line of the interstellar medium of star-forming galaxies. The $[\mathrm{C}$ 
II] and FIR luminosities are correlated, but the relation is non-linear. The $\mathrm{L}_{[\mathrm{CII}]} / \mathrm{L}_{\mathrm{FIR}}$ ratio is reduced by a factor of 20-50 with respect to lower luminosity star-forming galaxies, which is referred to as the "[C II] deficit." The $\mathrm{L}_{[\mathrm{CII}]} / \mathrm{L}_{\mathrm{FIR}}$ ratio correlates with the compactness of the mid-IR emitting region, and the [CII] deficit in most LIRGs is not caused by AGN activity but it is instead a fundamental property of the starburst itself. A primary conclusion is that [CII] is not a good SFR tracer in most local LIRGs, because it does not account for the increase in warm dust emission from the compact starburst.

Visual and Near-Infrared Image Analyses. Results of quantitative structural analysis of 85 (U)LIRGs with $\mathrm{L}_{\mathrm{ir}}>10^{11.4} \mathrm{~L}_{\odot}$ observed in B and I broadband filters with the Advanced Camera for Surveys (ACS) on the Hubble Space Telescope was presented by Kim et al. (2013). Derived mass ratios indicate that most of the systems involve major mergers, and the ratio of emission remaining in the residual images constructed from subtraction of smooth fits to the underlying galaxies indicates that the smaller companions suffer more tidal distortions than their larger companions. The results suggest that stellar bars are destroyed as mergers advance. Likewise, the fraction of light in the nuclei (unresolved AGN or nuclear starbursts) increases toward later merger stages and is highest in late-stage systems with a single nucleus. In addition, the subset of GOALS objects with elliptical-like hosts occupy the same portion of the surface brightness versus half-light radius plot (i.e., the "Kormendy Relation") and have similar slopes as nearby ellipticals in the Sloan Digital Sky Survey (SDSS), suggesting the possibility that the GOALS sources belong to the same parent population as the optically selected ellipticals.

Analysis of near-IR images acquired with the NICMOS instrument onboard HST for 73 GOALS objects with $\mathrm{L}_{\mathrm{ir}}>10^{11.4} \mathrm{~L}_{\odot}$ (Haan et al. 2011 reveals that $63 \%$ of the systems have double nuclei, and $6 \%$ may have triple nuclei. Half of the double nuclei unveiled at $1.6 \mu \mathrm{m}$ (H band) are not visible in HST B-band images due to obscuration by dust. The bulge luminosity surface density $\left(\mathrm{L}_{\mathrm{Bulge}} / \mathrm{R}^{2}\right)$ increases significantly along the merger sequence, and the bulge luminosity shows a slight increase in later stage mergers. The median projected nuclear separation is smaller for ULIRGs (1.2 kpc) than for LIRGs $(6.7 \mathrm{kpc})$, indicating that in most cases LIRGs occur earlier in the merger process than ULIRGs. A follow-up study of the HST H-band imagery (Haan et al. (2013)) shows that nuclear stellar cusps are resolved in $76 \%$ of the observed LIRGs, and the cusp strength and luminosity both increase with $\mathrm{L}_{\mathrm{ir}}$ (excluding AGN) and merger stage. Ultracompact nuclear starbursts are found in $13 \%$ of the LIRGs, and the nuclear near-IR surface density (measured within $1 \mathrm{kpc}$ radius) is found to increase by a factor of $\sim 5$ towards late merger stages. These results confirm theoretical models that predict starburst activity is linked to the build-up of stellar cusps in galactic nuclei (e.g., Hopkins et al. 2009).

Flux Ratios 8 SEDs. Comparison of the UV and IR properties of 135 GOALS objects observed by GALEX and Spitzer (Howell et al. 2010) showed that the contribution of far-UV radiation to the total measured SFR has a median of only $3 \%$ (ranging $0.2 \%$ to $18 \%)$, and the specific star formation rate (SSFR) has a median value $\left(3.9 \times 10^{10} \mathrm{yr}^{-1}\right)$ that is comparable to the highest SSFRs observed in the Spitzer Infrared Nearby Galaxies Survey (SINGS) sample. On average, the LIRGs have greater IR excesses than would be expected based on their UV colors if they obeyed the same scaling relations as normal star-forming galaxies with $\mathrm{L}_{\mathrm{ir}}<10^{11} \mathrm{~L}_{\odot}$. Only $21 \%$ of the resolved systems contain a single galaxy which dominates both wavelengths. A pilot study to derive the physical parameters of starbursts using spectral energy distribution (SED) fitting of 19 GOALS objects (Dopita et al. 2011) showed that the standard 'astronomical silicate' model does not provide a good fit to the silicate absorption features observed in the heavily dust-obscured starbursts. Using EVLA radio continuum observations in $1 \mathrm{GHz}$ windows 
centered at 4.7, 7.2, 29, and $36 \mathrm{GHz}$, Leroy et al. (2011) fit the radio SEDs of 22 (U)LIRGs in the GOALS sample and extended to higher frequency a previously observed deficit of high-frequency radio continuum emission for luminous starburst galaxies. Photometry performed in large apertures matched at radio through X-ray frequencies and results from fitting spectral energy distributions (SEDs) were presented by U et al. (2012) for 53 nearby LIRGs and 11 ULIRGs spanning $\log \left(\mathrm{L}_{\mathrm{ir}} / \mathrm{L}_{\odot}\right)=11.14-12.57$. They estimated $\mathrm{L}_{\text {ir }}, \mathrm{T}_{\mathrm{dust}}$, and $\mathrm{M}_{\mathrm{dust}}$ by fitting modified blackbodies to the mid-IR through sub-mm SEDs. Total stellar masses computed by fitting stellar population synthesis models to the observed near-IR through UV SEDs have a mean of $\log \left(\mathrm{M}_{*} / \mathrm{M}_{\odot}\right)=10.79 \pm 0.40$, and derived SFRs are $\sim 45 \mathrm{M}_{\odot} \mathrm{yr}^{-1}$ and $\sim 1.3 \mathrm{M}_{\odot} \mathrm{yr}^{-1}$ based on monochromatic IR and UV luminosities, respectively.

Dynamical Modeling. Results of dynamical modeling of four interacting systems were presented recently by Privon et al. (2013). Although two of the systems (the Mice and the Antennae) have been modeled previously in the literature, two additional systems (NGC $5257 / 8$ and NGC 2623) have been modeled for the first time using a consistent methodology that includes a single mass model and usage of the Identikit package (Barnes \& Hibbard 2009) to simplify matching of dynamical models to morphological and kinematic data for merging galaxies. Based on the assumed mass model and the derived initial conditions, the models reproduce the observed visual morphology and $\mathrm{H}$ I kinematics of the tidal tails quite well, and they indicate that the four systems are being viewed 175-260 Myr after first passage and thus cover a wide range of merger stages.

\section{Detailed Multiwavelength Studies of Individual Systems}

In addition to the survey results reviewed above, the wealth of data have provided the opportunity to conduct detailed studies of particularly interesting (U)LIRGs in the GOALS sample. Highlights of key findings published to date are reviewed here.

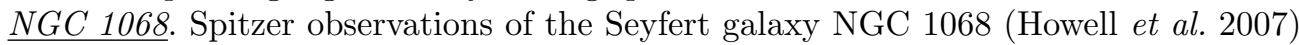
detected 8 and $24 \mu \mathrm{m}$ emission coinciding with star formation in the inner spiral $\sim 15^{\prime \prime}$ $(1 \mathrm{kpc})$ from the nucleus, and a bright complex of star formation located $\sim 47^{\prime \prime}(3 \mathrm{kpc})$ southwest of the nucleus. Strong PAH features at $6.2,7.7,8.6$, and $11.3 \mu \mathrm{m}$ are detected in IRS spectra measured in the vicinity of the inner spiral. The data rule out dust heated by the AGN as the primary emission source; their SEDs are dominated by starlight and $\mathrm{PAH}$ emission. The brightest $\mathrm{PAH}$ and $24 \mu \mathrm{m}$ emitting regions are located precisely where two spiral arms of molecular gas emerge from the ends of the inner stellar bar; this is consistent with kinematic models that predict gas gets trapped within the inner Lindblad resonance of a large stellar bar which itself contains a smaller, weaker bar.

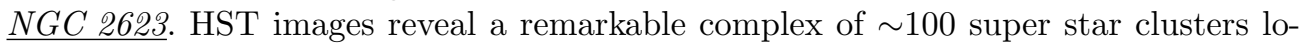
cated predominantly in a region that extends over $3 \mathrm{kpc}$ south of the nucleus in the advanced merger system NGC 2623 (Evans et al. 2008). The star clusters have absolute magnitudes similar to those observed in the well-studied Antennae galaxies, and comparison of the broadband colors with population synthesis models indicates ages of 1-100 Myr. Detection of the [Ne v] $14.32 \mu \mathrm{m}$ emission line confirms evidence from X-ray and radio data that an AGN is present. The off-nuclear starburst is estimated to account for less than $1 \%$ of the current star formation in the system, with the majority of the activity being heavily obscured at UV and visual wavelengths.

$\underline{I I ~ Z w ~ 96}$. Spitzer Space Telescope observations of the interacting galaxy system II Zw 96 by Inami et al. (2010) reveal a dramatic example of how the appearance of (U)LIRGs is strongly dependent on the observed wavelength region. While images from the HST in the visual (B and I bands) show what appears to be two galaxies, images from Spitzer 
at 3.6 and $8.0 \mu \mathrm{m}$ reveal a third bright infrared source. The $24 \mu \mathrm{m}$ image is completely dominated by the third source that has no obvious counterpart at visual wavelengths and is responsible for the bulk of the infrared luminosity of the system, which is nearly a ULIRG $\left(\mathrm{L}_{\mathrm{ir}}=10^{11.9} \mathrm{~L}_{\odot}\right)$. If this is an off-nuclear starburst, it is about 100 times more luminous than the corresponding off-nuclear infrared source in the Antennae Galaxies, with a corresponding SFR of $\sim 120 M_{\odot} y r^{-1}$. An alternative hypothesis is that the dominant infrared source is a heavily obscured nucleus of a triple galaxy system.

Mrk 273. In the ULIRG Mrk 273, Iwasawa et al. (2011) found that a highly absorbed hard X-ray source detected by Chandra is coincident with the SW near-IR nucleus (a confirmed AGN), a bright off-nuclear radio continuum source is co-spatial with the brightest star cluster detected in HST imagery, and a large nebula detected in soft X-rays contains a dark lane that spatially coincides with a high surface-brightness tidal tail extending $\sim 50^{\prime \prime}(40 \mathrm{kpc})$ south of the galaxy. The column density of cold gas absorbing the soft X-rays is $\mathrm{N}_{\mathrm{H}} \geqslant 6 \times 10^{21} \mathrm{~cm}^{-2}$, consistent with an edge-on orientation of the tidal tail.

$\underline{I C ~ 883}$. A multiwavelength study of the late-stage merger IC 883 (Modica et al. 2012) revealed 156 star clusters located primarily in the circumnuclear region $(3-7 \mathrm{kpc}$ from the mid-IR core) and throughout the tidal tails. The clusters have a luminosity function similar to clusters in other star-forming galaxies, and the broadband colors are consistent with a starburst population synthesis model with ages of approximately $10^{7}-10^{9} \mathrm{yr}$, or as young as a few Myr considering the (unknown) extinction. Detection of the [Ne v] $14.32 \mu \mathrm{m}$ emission line indicates an AGN is present; however, the weakness of the [Ne v] line and the small equivalent width of the $6.2 \mu \mathrm{m}$ PAH feature suggest the AGN plays an insiginificant role in heating the dust in this starburst-dominated system.

Mrk 266. An in-depth investigation of Mrk 266 (Mazzarella et al. 2012) resulted in numerous findings including confirmation of dual active nuclei, evidence for a Comptonthick X-ray source in the SW nucleus, a radiative bow shock in an outflow surrounding the NE nucleus, shock-heated gas between the nuclei, detection of $24 \mu \mathrm{m}$ co-spatial with the prominent Northern Loop detected in optical emission-lines, radio continuum and soft X-rays which suggests that $\sim 2 \times 10^{7} M_{\odot}$ of dust is entrained in an outflowing superwind, 120 young star clusters, and a substantial fraction of the total CO (1-0) line emission bridging the two galaxies. Comparison of observed properties with numerical simulations of major mergers, along with the total molecular gas mass, suggest Mrk 266 may evolve into a ULIRG. It is proposed that rapid gas consumption in luminous dual AGN early in the merger process (kpc-scale separations) may explain the paucity of detected binary QSOs (parsec-scale orbital separations) in spectroscopic surveys, and an evolutionary sequence is proposed representing a transition from dual to binary AGN with a corresponding increase by a factor of 100 or more in the observed $L_{x} / L_{i r}$ ratios.

NGC 34. ALMA Cycle-0 observations of the advanced merger system NGC 34 (Xu et $\overline{\text { al. } 2014}$ ) show that the CO (6-5) line emission is well resolved by the small ALMA beam $\left(0.26^{\prime \prime} \times 0.23^{\prime \prime}\right)$, revealing a nearly face-on rotating disk of radius $\sim 400 \mathrm{pc}$, and the $435 \mu \mathrm{m}$ dust continuum is concentrated in a region more compact than the gas disk. Both the CO (6-5) line and the dust continuum spatial distributions suggest a very high gas column density $\left(\sim 10^{4} \mathrm{M}_{\odot} \mathrm{pc}^{-2}\right)$ in the nuclear region (radius $<100 \mathrm{pc}$ ).

Arp 220. High-resolution 6 and $33 \mathrm{GHz}$ EVLA observations of the ULIRG Arp 220 shows $6 \mathrm{GHz}$ continuum emission that is co-spatial with $\mathrm{CO}(3-2)$ gas emission, and the $33 \mathrm{GHz}$ continuum emission (FWHM $=8$ mas) reveals exponential disks surrounding both nuclei that trace quite well the surface density of radio supernovae observed with VLBA observations (Barcos-Munoz et al. 2014, in preparation). 


\section{Work in Progress}

We have only touched on highlights of results published to date from the GOALS program, and readers are encouraged to refer to the cited articles for further results that are increasing our understanding of (U)LIRGs in the local universe. Work in progress includes further analysis of the Spitzer and Herschel imaging and spectroscopic data, additional interferometry with the JVLA and ALMA to probe the physical conditions in the nuclear regions, and dynamical simulations of a much larger sample to make further progress in quantitative age dating of merger events and correlating the dynamical states with observations and derived physical properties to improve our understanding of the evolution of merger-induced starbursts and AGN.

\section{Acknowledgements}

The GOALS team includes L. Armus, P. Appleton, J. Barnes, G. Bothun, B. Chan, A. S. Evans, D. T. Frayer, S. Haan, J. H. Howell, H. Inami, K. Iwasawa, L. J. Kewley, D.-C. Kim, S. Lord, B. F. Madore, J. Marshall, J. M. Mazzarella, E. J. Murphy, A. Petric, G. C. Privon, J. Rich, D. B. Sanders, B. Schultz, H. Spoon, E. Sturm, S. Stierwalt, J. A. Surace, V. U, T. Vivilkin, S. Veilleux, and C. K. Xu. Please consult the cited articles for a complete list of co-authors and affiliations. NASA support is gratefully acknowledged.

\section{References}

Armus, L., Mazzarella, J. M., Evans, A. S. et al. 2009, PASP, 121, 559

Barnes, J. E. \& Hibbard, J. E. 2009, AJ, 137, 3071

Caputi, K. I., Lagache, G., Yan, Lin et al. 2007, ApJ, 660, 97

Daz-Santos, T. and Charmandaris, V. and Armus, L. et al. 2010, ApJ, 723, 993

Daz-Santos, T., Charmandaris, V., Armus, L. et al. 2011, ApJ, 741, 32

Daz-Santos, T., Armus, L, Charmandaris, V. et al. 2013, ApJ, 774, 68

Dopita, M. A., Armus, L., Kewley, L. J. et al. 2011, Ap\&SS, 333, 225

Evans, A. S., Vavilkin, T., Pizagno, J. et al. 2008, ApJ (Letters), 675, L69

Haan, S., Surace, J. A., Armus, L. et al. 2011, AJ, 141, 100

Haan, S., Armus, L., Surace, J. A. et al. 2013, MNRAS, 434, 1264

Hopkins, P. F., Lauer, T. R., Cox, T. J. et al. 2009, ApJS, 181, 486

Howell, J. H., Mazzarella, J. M., Chan, B. H. P.. et al. 2007, AJ, 134, 2086

Howell, J. H., Armus, L., Mazzarella, J. M. et al. 2010, ApJ, 715, 572

Inami, H., Armus, L., Surace, J. A. et al. 2010, AJ, 140, 63

Iwasawa, K., Sanders, D. B., Evans, A. S. et al. 2009, ApJ (Letters), 695, L103

Iwasawa, K., Sanders, D. B., Teng, S. H. et al. 2011, A\& A, 529, A106

Iwasawa, K., Mazzarella, J. M., Surace, J. A. et al. 2011, A\&A, 528, A137

Kim, D.-C., Evans, A. S., Vavilkin, T. et al. 2013, ApJ, 768, 102

Le Floc'h, E., Papovich, C., Dole, H. et al. 2005, ApJ, 632, 169

Leroy, A. K., Evans, A. S., Momjian, E. et al. 2011, ApJ (Letters), 739, L25

Mazzarella, J. M., Iwasawa, K., Vavilkin, T. et al. 2012, AJ, 144, 125

Modica, F., Vavilkin, T., Evans, A. S., et al. 2012, AJ, 143, 16

Petric, A. O., Armus, L. A., Howell, J. et al. 2011, ApJ, 730, 28

Privon, G. C., Barnes, J. E., Evans, A. S. et al. 2013, ApJ, 771, 120

Sanders, D. B., Mazzarella, J. M., Kim, D.-C. et al. 2003, AJ, 126, 1607 (RBGS)

Sanders, D. B. \& Mirabel, I. F. 1996, ARAA, 34, 749

Stierwalt, S., Armus, L., \& Surace, J. A. et al. 2013, ApJS, 206, 1

U, Vivian, Sanders, D. B. Mazzarella, J. M. et al. 2012, ApJS, 203, 9

Veilleux, S. 2006, New Astron. Revs, 50, 701

Xu, C. K., Cao, C., Lu, N. et al. 2014, ApJ, submitted 